\title{
Note stylistique sur le récit de voyage : les pages bayonnaises de Pyrénées (1843) de Victor Hugo
}

\author{
André LABERTIT \\ Université de Strasbourg \\ orfelunio75@gmail.com
}

Recibido: $29 / 03 / 2016$

Aceptado: 27/09/2016

Résumé

Cette étude sur les pages bayonnaises extraites de Pyrénées (1843) s'inscrit dans une recherche sur la nature littéraire des récits de voyages et son expression stylistique. Écrites 32 ans après une étape à Bayonne sur le chemin qui conduisait Victor Hugo, enfant, auprès de son père à Madrid, elles sont considérées dans leur singularité organique. Le moi de Hugo, tout imprégné qu'il est de Bayonne, de l'Espagne, de Napoléon, envahit la relation référentielle ; le temps remémoré ne l'est pas en vue de retrouver le passé mais de l'évoquer dans une rêverie moins divagante qu'orientée. Évocation proprement créatrice, rendue patente par l'écart entre le descriptible et le décrit. La méthode adoptée s'attache au contenu du texte (onomasiologie) et à sa réception (sémasiologie).

Mots clés : Hugo voyageur, stylistique du récit de voyage, Pyrénées, Bayonne, Espagne.

\section{Nota estilística sobre el relato de viajes: \\ las páginas de Bayona en Pyrénées (1843) de Víctor Hugo}

\section{Resumen}

Este estudio acerca de las páginas bayonesas extraídas de Pyrénées (1843) forma parte de una investigación sobre la naturaleza literaria de los relatos de viajes y de su expresión estilística. Escritas 32 años después de una estancia en Bayona, que formaba parte del camino que llevaba a Víctor Hugo, todavía niño, en un viaje hacia Madrid junto a su padre, son consideradas en su singularidad orgánica. El yo de Hugo, absolutamente impregnado de Bayona, de España, de Napoleón, invade la relación referencial; el tiempo recordado no toma forma de recuperación, sino de evocación de una ensoñación menos divagante que orientada. Una evocación que es completamente creadora, patente sobre todo por la distancia entre lo descriptible y lo descrito. El método adoptado se atiene al contenido del texto (onomasiología) y a su recepción (semasiología).

Palabras clave: Hugo viajero, estilística del relato de viajes, Pyrénées, Bayona, España. 


\title{
On the Style of Travel Narratives: The Bayonne pages from Victor Hugo's Pyrénées (1843)
}

\begin{abstract}
This study of the pages that Victor Hugo devoted to Bayonne in his Pyrénées (1843) is part of research conducted into the literary nature and style of travel narratives. Written thirty-two years after his stay in Bayonne-which was one of the stops on a trip to Madrid that Hugo, still a kid, made in the company of his father - these pages are analyzed in their organic singularity. Hugo's self, absolutely impregnated with Bayonne, Spain, and Napoleon, pervades all referential signs in the novel. His memories do not take the shape of something recovered, but they evoke instead an insinuated reverie. This is an ostensibly creative evocation, discernible in the distance that exists between the describable and the described. Our method considers the contents of the text (onomasiology) as well as its reception (semasiology).
\end{abstract}

Keywords: Hugo traveler, stylistics of the travel stories, Pyrénées, Bayonne, Spain.

\section{Referencia normalizada}

Labertit, A. (2016). « Note stylistique sur le récit de voyage : les pages bayonnaises de Pyrénées (1843) de Victor Hugo ». Thélème. Revista Complutense de Estudios Franceses, Vol. 31, Núm. 2 : 271-281. http://dx.doi.org/10.5209/rev_THEL.2016.v31.n2.52175

Le 18 juillet 1843, Victor Hugo quitte Paris. Juliette Drouet l'accompagne. Il entreprend un voyage qui le conduit en Aquitaine. Je l'arrête à Bayonne. Je dispose d'un texte (Pyrénées) entièrement rédigé et apparemment prêt pour l'impression. Il ne verra pas le jour du vivant de l'auteur. Je l'emprunte à l'édition des Euvres complètes, volume Voyages, édition Bouquins, 1987. Toutes les références y renvoient. Hugo l'a écrit à Bayonne les 26 et 27 juillet 1843. À regret, j'en retire les pages, également écrites à Bayonne, consacrées à Biarritz et à la visite du charnier Saint Michel à Bordeaux.

Quelles sont mes assises face à un texte de Hugo, qui en 1843, n'en est pas à son premier voyage ni à son premier récit de voyage ?

Primo, son besoin et son plaisir irrépressibles d'écrire, où qu'il se trouve. L'anecdote du pont de Dax - à laquelle je me permets de renvoyer (Daru, $1961: 471$ 474) - le montre à souhait : récit et événement y relèvent de deux ordres distincts, le monde du voyage n'est pas que spéculaire. Secundo, le but du voyage : «Ce ne sont pas des évènements que je cherche en voyage, ce sont les idées et les sensations», répète sans cesse Hugo (Le Rhin, Lettre XX). L'imagination est la plus forte. Elle supplée le spectacle. La rêverie emplit l'esprit (Hugo, 1987 : 761). Tertio, le secret du voyage : il n'est pas dans le voyage. Il n'est connu que des « fantômes ", ceux du charnier de Saint Michel, par exemple (Hugo, 1987: 772). On lit dans « À Villequier », vers 41 : « Nous ne voyons jamais qu'un seul côté des choses / L'autre plonge en la nuit d'un mystère effrayant ». Quarto, Bayonne : «Bayonne est restée 
dans mon cœur comme un lieu vermeil et souriant. C'est là qu'est le plus ancien souvenir de mon cœur» (Hugo, $1987: 766$ ).

Ces données rappelées, suffira-t-il de recentrer le récit sur son foyer le plus intime, de parler de " voyage égotiste ou, flâneusement s'entremêlent les routes et les rêves » ? (Gély, 1993 : 239). Certaines opinions critiques, dont de Claude Gély elle-même, font état de "réminiscences», de «subtils amalgames » présents dans des fictions narratives, dans des poèmes écrits ultérieurement aux récits de voyage, le tout lié à des « rencontres de la route, à des rêveries au fil des anciens voyages » (Gély, 1993 : 240). Existerait-il une solidarité profonde entre tel passage des Voyages et tel épisode romanesque ou tel poème ? Stimulantes remarques. Elles sont à reconsidérer.

Victor Hugo a menti à sa chère Léopoldine et aux siens quand il leur écrit de Bayonne : « Je suis venu de La Rochelle ici par mer et comme je le marque à ta mère, en arrivant à Biarritz, j'ai lu dans les journaux que j'étais à Bordeaux et dans d'autres que j'étais en Suisse » (Hugo, 1987 :1266). Que signifie ce brouillage ? D'autant que dans le récit nous lisons : " Je n'ai pu entrer à Bayonne sans émotion. Bayonne est pour moi un souvenir d'enfance. Je suis venu à Bayonne étant tout petit ayant sept ou huit ans, vers 1811 ou 1812, à l'époque des grandes guerres » (Hugo, $1987: 763$ ).

Ici, les érudits sont à la fête. Ils peuvent reprendre Hugo sur ses inexactitudes : c'est en effet en 1811, quand il avait neuf ans, qu'avec sa mère et ses frères, Abel et Eugène, il séjourna à Bayonne un mois durant avant d'aller rejoindre le général Hugo à Madrid. Ces inexactitudes ont donné lieu à des interprétations. La précision datée, Victor Hugo, à ce qu'il parait, s'en soucie comme d'une guigne. Il lui arrive bien de vaciller quelquefois sur une date, un événement, un lieu. Cela se voit à une apostille. Ici il n'y en a point.

Bayonne habite depuis longtemps le poète. Deux odes, déjà, y renvoyaient : «À mon père» et «Mon enfance». Elles indiquent que Bayonne et l'Espagne se confondent dans un même souvenir. Sainte-Beuve corrobore : « Il avait surtout dans l'imagination ces graves et hauts souvenirs d'enfance qui lui ont imprimé, comme on l'a dit ici heureusement, un pli [...] grandiose » (Sainte-Beuve, $1885: 454)$. On peut croire Sainte-Beuve.

En cet été de 1843, Victor Hugo revient à Bayonne. Par où commence-t-il sa relation, une fois sur les lieux? Par ses souvenirs, justement. Il se délecte de réminiscences.

Je me rappelle que le lendemain de notre arrivée à Bayonne une espèce de signor ventru, orné de breloques exagérées et baragouinant l'italien, se présenta chez ma mère. Cet homme nous fit, à nous enfants qui le regardions entrer à travers les portes vitrées l'effet d'un charlatan de place. C'était le directeur du théâtre de Bayonne. Il venait prier ma mère de prendre une loge à son théâtre. Ma mère loua une loge pour un mois. C'était à peu près le temps que nous devions rester à Bayonne. Cette loge louée nous fit sauter de joie. Nous enfants, aller au spectacle tous les soirs pendant tout un mois, nous qui n'étions entrés dans un théâtre qu'une fois par an, et qui n'avions dans l'esprit d'autre souvenir dramatique que La Comtesse d'Escarbagnas. Le soir même nous tourmentâmes ma mère, qui nous obéit, comme les mères font toujours, et nous mena au théâtre. Le contrôleur nous installa dans une magnifique loge de face ornée de draperie de calicot rouge à rosaces safran. On jouait Les Ruines de Babylone, fameux mélodrame qui avait en ce temps-là un immense succès dans toute la France. C'était 
magnifique, à Bayonne du moins. [...] Nous étions dans l'admiration. Le lendemain, le soir venu, nous tourmentâmes encore notre mère qui nous obéit encore. Nous voici au spectacle dans notre loge à rosaces. - Que va-t-on donner ? Nous étions dans l'anxiété. La toile se lève [...] On donnait Les Ruines de Babylone [...] Cela dura ainsi tout le mois. Un beau jour l'affiche changea. Ce jour-là nous partions. C'est ce souvenir-là qui m'a fait parler quelque part de ce hasard taquin qui joue avec l'enfant (Hugo, $1987: 764)$.

L'évocation de la maison suit : « La maison que nous habitions était riante. Je me rappelle ma fenêtre où pendaient de belles grappes de maïs mûr. Pendant tout ce long mois, nous n'eûmes pas un moment d'ennui ; j'excepte toujours Les Ruines de Babylone » (Id.). La maison, Hugo ne la retrouve pas. Tant bien que mal il la relocalisera, quelque part sur les remparts.

Ici encore, les commentateurs ont noté que la narration diffère sensiblement des descriptions que donne Victor Hugo raconté par un témoin de sa vie et Victor Hugo raconté par Adèle Hugo. Pour moi, je ne regarde pas du côté des divergences, je vais aux convergences, aux relations entre textes.

Ce qui suit, les jeux avec les frères, les barbouillages, les gravures d'un exemplaire des Mille et une nuits - don du général Lahaurie mort fusillé à la plaine de Grenelle ici l'érudition est secourable et permet d'annoter le texte, complète la relation (Hugo, 1985 : 1270). Je passe... Ce flot mémoriel se clôt - preuve qu'il est organisé -. Une opportune transition le relance : la description de la logeuse qui, à son tour, va servir de transition à l'un des deux épisodes majeurs relatés dans ces pages : "C C'était une personne de la ville, une veuve, je crois, qui louait cette maison à ma mère » (Hugo, 1987 : 765). Et voici le morceau fameux :

Elle avait une fille de quatorze ou quinze ans. Ma mémoire après trente années n'a perdu aucun des traits de cette angélique fille. Je la vois encore. Elle était blonde et svelte, et me paraissait grande. C'était un regard doux et voilé, un profil virgilien, comme on rêve Amaryllis ou la Galathée qui s'enfuit vers les saules. Elle avait le cou admirablement attaché et d'une pureté adorable, la main petite, le bras blanc et le coude un peu rouge, ce qui tenait à son âge, détail que le mien ignorait alors. Elle était habituellement coiffée d'un madras thé à bordure verte étroitement serré du sommet de la tête à la nuque, de façon à laisser le front à découvert et ne cacher que la moitié de la chevelure. Je ne me rappelle pas la robe qu'elle portait (Hugo, $1987: 765$ ).

Le dernier trait est d'un maître. Il sert de garant à tous les autres. Nous sommes mis en présence d'une jeune fille qualifiée d' « angélique », de « virgilienne » - ici encore l'érudition est secourable : Bucoliques, III, v. 64-65. Suggestion littéraire et souvenir s'épaulent non sans un subtil balancement suscité tactiquement par 1'indécision. Peut-être était-elle, la jeune fille, alors comparable aux figures mythologiques des livres du jeune Hugo - mais à qui aurait-il pu autrement la comparer ? - ou telle qu'il se la représente maintenant : une promesse d'amour et la déception de cette promesse, Galathée « qui veut se faire voir avant de disparaître sous les saules» (Et fugit at salices, et se cupit ante videre). "Virgilien », « angélique » sont deux épithètes de nature, c'est-à-dire des outils de langage parfaitement aptes à expliciter l'implicite par référence au savoir érudit. Chacune a pour fonction canonique de désigner un type de pureté et de beauté. Par elles-mêmes, 
elles ne sont pas descriptives comme le seraient des adjectifs pris en dehors du référent mythologique, elles ne produisent aucun effet de réel. Il faut en conclure que Victor Hugo, ici, ne donne pas à voir son personnage mais à le reconnaître selon la figure qu'il en a formée pour lui-même, qu'il en a gardée ou réarrangée dans son souvenir. Après le portrait, la scène :

Cette belle enfant venait jouer avec nous. Quelquefois Abel et Eugène, mes aînés, plus grands et plus sérieux que moi et «faisant les hommes », comme disait ma mère allaient voir l'exercice à feu sur le rempart ou monter dans leurs chambres pour étudier Sobrino et feuilleter Cormon. Alors j'étais seul, je sentais l'ennui venir, que faire ? Elle m'appelait, et me disait : viens que je te lise quelque chose. Il y avait dans la cour une porte exhaussée de quelques marches et fermée d'un gros verrou rouillé que je vois encore, un verrou rond, à poignée en queue de porc comme on en voit dans les vieilles caves. C'était sur ces marches qu'elle allait s'asseoir. Je me tenais debout derrière elle le dos appuyé à la porte. Elle me lisait je ne sais plus quel livre ouvert sur ses genoux. Nous avions au-dessus de nos têtes un ciel éclatant et un beau soleil qui pénétrait de lumière les tilleuls et changeait les feuilles vertes en feuilles d'or. Un vent tiède passait à travers les fentes de la vieille porte et nous caressait le visage. Elle était courbée sur son livre et lisait à voix haute. Pendant qu'elle lisait, je n'écoutais pas le sens de ses paroles, j'écoutais le son de sa voix. Par moments, mes yeux se baissaient ; mon regard rencontrait son fichu entr'ouvert au-dessous de moi, et je voyais avec un trouble mêlé d'une fascination étrange sa gorge ronde et blanche qui s'élevait et s'abaissait doucement dans l'ombre, vaguement dorée d'un chaud reflet du soleil. Il arrivait parfois dans ces moments-là qu'elle levait tout à coup ses grands yeux bleus et elle me disait : eh bien,Victor! Tu n'écoutes pas? J'étais tout interdit, je rougissais et je tremblais et je faisais semblant de jouer avec le gros verrou (Hugo, 1987 : 765).

Il n'échappe pas à Victor Hugo, quoi qu'il en dise, que la scène n'est pas totalement idyllique : «O époque naïve et pourtant doucement agitée. C'est là que j'ai vu poindre dans le coin le plus obscur de mon âme, cette première lueur inexprimable, aube divine de l'amour » (Hugo, 1987: 766). La scène est relatée en ce qu'elle avait d'itératif, l'imparfait y pourvoyant. C'est le temps de l'itération, c'est aussi le temps des fables.

Après cette ample analepse, le récit fait place au commentaire : « Ne trouvez-vous pas, ami, que pareil souvenir ait un lien et un lien que rien ne peut détruire ?» (Ibid.) Faux dialogue. Le genre oblige, Hugo s'interroge lui-même : "Chose étrange que deux êtres puissent être liés de cette chaîne toute la vie et ne pas se manquer pourtant, et ne pas se chercher, et être étranger l'un à l'autre et ne pas même se connaître ! La chaîne qui m'attache à cette douce enfant ne s'est pas rompue mais le fil s'est brisé » (Hugo, 1987 : 766).

Suivent ces questions : «Où est-elle ? Que fait-elle? Est-elle morte ? Vit-elle encore ? » Questions sans réponse, évidemment, et reprise du thème archi-classique de l'ubisunt. Dans le maintenant du récit, Victor Hugo dresse ce constat: «La mémoire, ce point du passé, est brisée entre elle et moi, elle ne connaîtrait pas mon visage et je ne reconnaitrai pas le son de sa voix. Elle ne sait plus mon nom et je ne sais pas le sien » (Ibid.)

Surprenante assertion! En rigueur, qu'en sait Hugo ? Que sait-il d'elle ? Force est de considérer que les questions métaphysiques ouvertes par l'ubisunt avaient une couleur rhétorique : Hugo connaissait les réponses. J'y reviendrai. 
C'est à propos de ces pages que la critique contemporaine a parlé de «traces », d'« amalgames ». Dans l'Art d'être grand-père, effectivement, un poème intitulé « Pepita » retient l'attention. Daté du 16 janvier 1855 il occupe à lui seul la section IX du recueil intitulé «Les fredaines du grand-père enfant (1811) ». Nous sommes revenus à cette époque. Mais ce texte reporte vers l'enfance à Madrid et non à Bayonne. Victor Hugo raconté par un témoin de sa vie donne cette précision : « Il se trouvait là une nommé Pepita, encore petite fille. [...] Son père était le marquis de Monte Hermosa. [...] Pepita était de leurs parties au jeu de cache-cache, se mettait dans les grands vases avec eux. Elle avait trois cavaliers servants empressés. Il y eut des idylles, me disait mon mari » (Hugo, $1985: 126$ ).

La demoiselle n'est pas une invention littéraire. La critique croit la retrouver dans "Vieilles chansons du jeune temps », poème présent dans Les contemplations (I, XIX). Un autre rattachement pourrait être fait, à meilleur droit, avec le poème « Lise » du même recueil (I, XII) dont voici le début :

\author{
J'avais douze ans ; elle en avait bien seize. \\ Elle était grande et moi, j'étais petit. \\ Pour lui parler le soir plus à mon aise, \\ Moi j'attendais que sa mère sortît ; \\ Puis je venais m'asseoir près de sa chaise \\ Pour lui parler le soir plus à mon aise (Hugo, 1995 : 45).
}

Poème et récit de voyage sont contemporains. Le prénom de la jeune fille est absent du récit de voyage. Lise, celui de la jeune fille du poème, n'est pas sans relation avec l'action de la scène bayonnaise : une scène de lecture. Le texte dit :

Elle m'aimait. Je l'aimais. Nous étions

deux purs enfants, deux parfums, deux rayons (Id.).

Je récapitule: détails mis à part et distinction faite entre les différentes scénographies, dans ces textes, on trouve une jeune fille espagnole ou rencontrée sur le chemin de l'Espagne auprès de qui le jeune Hugo éprouve ses premiers émois amoureux. Le motif, lui, est stable. J'y vois la preuve que Bayonne et l'Espagne sont à jamais associées chez Hugo à la découverte de l'amour. Considéré de la sorte, l'épisode bayonnais, s'inscrit dans une transtextualité.

Retour au texte. La description de Bayonne est très réduite. Victor Hugo s'en rend compte et le confesse : «J'ai peu à vous dire de Bayonne» (Hugo, 1987: 766). De fait, il y consacre à peine quelques lignes : « Bayonne, jolie ville et beau lieu ». Elle serait un pur chef-d'œuvre du Bon Dieu si Vauban et sa citadelle ne l'avait gâtée. Victor Hugo n'aime que les paysages intacts. Ensuite il visite la cathédrale (Hugo, 1987 : 767). Une fois de plus, la «sensation » éprouvée l'emporte. Hugo est plein d'admiration pour l'édifice qu'il compare à ceux de Chartres et de Reims. Malgré tout, c'est peu.

C'est qu'il lui faut courir à Biarritz où tout le monde court. Le site l'émerveille. Il le jauge par rapport à ce qu'il admire ailleurs : « Les trois points de la côte normande 
qui m'agréent le mieux, le bourg d'Ault, le Tréport et Étretat» (Hugo, 1987 : 777). Je passe et reviens à Bayonne.

Nous sommes le 27 juillet. Il est tard. Hugo rentre en voiture de Biarritz. Dans la voiture qui le ramène, un « compagnon de voyage », raconte-t-il, lui « montre dans l'ombre sur une colline » le château de Marrac - graphie gasconne - « ou du moins ce qu'il en reste » (Hugo, 1987 : 778). C'est une transition-jointure pour une seconde analepse greffée sur le récit. Au premier abord, elle est tout « externe» pour la qualifier en termes d'analyse du récit, totalement « hétérodiégétique ». Que signifiet-elle? Une ouverture, elle aussi, des vannes du passé qui vont laisser place - je ne dis pas libre cours - au second flot de souvenirs attachés à Bayonne: ceux de l'époque napoléonienne, l'époque des "grandes guerres » comme Hugo disait en commençant d'écrire ces pages (Hugo, 1987 : 763). Cette fois encore, il ne décrit pas. Il rêve :

Le château de Marrac est célèbre pour avoir été en $1808^{* 1}$ le logis de l'empereur à l'époque de l'entrevue de Bayonne. Napoléon avait en cette occasion une grande pensée ; mais la providence ne l'accepta pas ; et quoique Joseph Ier ait gouverné les Castilles comme un bon et sage prince, l'idée, si utile pourtant à l'Europe, à la France, à l'Espagne et à la civilisation, de donner une dynastie neuve à l'Espagne fut funeste à Napoléon comme elle l'avait été à Louis XIV. Joséphine qui était créole et superstitieuse, accompagnait l'empereur à Bayonne. Elle semblait avoir je ne sais quels pressentiments, et comme Nunéz Saledo dans la romance espagnole, elle répétait souvent : il arrivera malheur de ceci. (Hugo, 1987 : 778).

Au vrai, Hugo dérive selon son erre. Le mouvement de la voiture y aide. Il va droit au symbole et l'objective : " Aujourd'hui qu'on voit le revers de ces événements déjà enfoncés dans l'histoire à une distance de trente années, on distingue, dans les moindres détails, tout ce qu'ils ont eu de sinistre, et il semble que la fatalité en ait tenu tous les fils» (Hugo, 1987 : 779). Marrac symbolise l'avenir des événements dont, «à distance de trente ans " et "déjà enfoncés dans l'histoire », chacun - l'objectivation - peut voir le «revers» et distinguer la cause : la «fatalité ». Transporté dans le passé, ou s'y transportant, Hugo le charge de menaces que le futur confirmera. C'est facile, mais le tour lui permet d'installer Joséphine, l'impératrice, sur le devant du récit, en prophétesse des malheurs à venir.

Deuxième temps, non moins analytique, justifié sous raison de verser aux dossiers de l'histoire « une particularité tout à fait inconnue », Victor Hugo ajoute :

Pendant son séjour à Bayonne, l'empereur voulut visiter les travaux qu'il faisait exécuter au Boucaut. Les bayonnais qui avaient alors âge d'homme se souviennent que l'empereur un matin traversa à pied les allées marines pour aller gagner le brigantin mouillé dans le port qui devait le transporter à l'embouchure de l'Adour. Il donnait le bras à Joséphine. Comme partout il avait là sa suite de rois, et dans cette conjoncture c'étaient les princes du midi et les Bourbons d'Espagne qui lui faisait cortège, le vieux roi Charles IV et sa femme, le prince des Asturies qui depuis a été roi et s'est appelé Ferdinand VII ; don Carlos, aujourd'hui prétendant sous le nom de Charles V. Toute la population de Bayonne

${ }^{1}$ L'astérisque renvoie à la mention « vérifier ». 
était dans les allées marines et entourait l'empereur qui marchait sans gardes. Bientôt la foule devint si nombreuse et si importune dans sa curiosité méridionale que Napoléon doubla le pas. Les pauvres Bourbons, essoufflés le suivaient à grand peine. L'empereur arriva au canot du brigantin d'une marche si précipitée qu'en y entrant Joséphine, voulant saisir en hâte la main que lui tendait le capitaine du navire, tomba dans l'eau jusqu'aux genoux. En toute autre circonstance elle n'aurait fait qu'en rire. C'eut été pour elle, me disait en me contant la chose madame la duchesse de $\mathrm{C}^{* * *}$, une occasion de montrer sa jambe qu'elle avait charmante. Cette fois, on remarqua qu'elle secoua la tête tristement (Hugo, 1987 : 779).

Cette anecdote proviendrait d'une conversation privée. Victor Hugo serait-il soucieux de protéger son informatrice ? La chasse est ouverte, la chasse aux sources. Elle est très aventureuse tant que les sourciers ignorent où Hugo les puise. À ma connaissance, avant Victor Hugo, seul Jean-Baptiste Bailac, auteur des Nouvelles chroniques de la ville de Bayonne par un Bayonnais, 1827, relate des faits apparentés. Napoléon et Joséphine, nous dit Bailac, aimaient se rendre sur la plage du Boucau pendant leur séjour à Bayonne. Ils jouaient sur la grève. Un jour, « d'un air folâtre », Napoléon aurait poussé l'impératrice dans l'eau jusqu'à mi-jambe (Bailac, $1827: 327)$.

Faisons le point: premièrement, l'épisode est mis par Hugo au compte de « bayonnais qui avaient âge d'homme ». C'est le cas de Bailac (1768-1848). Mais l'indication n'est guère plus garantie que celle donnée par une duchesse anonyme. Deuxièmement, il n'a rien à voir avec les folâtreries du couple impérial sur la plage du Boucau - ou de la Barre. Troisièmement, la course des «Bourbons » haletants derrière un Napoléon qui presse le pas, a un petit air de connu. Je veux dire de motif connu : Mayenne courant derrière Henri IV, par exemple. Alors, anecdote ou motif narratif? Mais convient-il de s'interroger sur l'authenticité de l'épisode?

Le commentaire qui suit développe à nouveau le thème des "pressentiments " introduit quelques lignes plus haut, quand il était question de Marrac. C'est un indice fort dont il faut donner raison. Celle-ci est fonctionnelle, elle porte sur la chute de l'Empire et l'explication qu'en donne Victor Hugo. Ce n'est donc pas l'authenticité de l'épisode qui me parait ici devoir être dégagée mais sa fonctionnalité. D'autant que le texte qui suit renchérit :

Le présage était mauvais. Tout ce qui assistait à cette aventure a fait une triste fin. Napoléon est mort proscrit ; Joséphine est morte répudiée ; Charles IV et sa femme sont morts détrônés ; quant à ceux qui étaient alors de jeunes princes, l'un est mort, Ferdinand VII ; l'autre, don Carlos, est prisonnier. Le brigantin qu'avait monté l'empereur s'est perdu deux ans après corps et biens sous le cap Ferret dans la baie d'Arcachon; le capitaine qui avait donné la main à l'impératrice, et qui s'appelait Lafon, a été condamné à mort pour ce fait, et fusillé. Enfin le château de Marrac, où Napoléon avait logé, transformé successivement en caserne et en séminaire, a disparu dans un incendie. En 1820, pendant une nuit d'orage, une main, restée inconnue, y mit le feu aux quatre coins (Id.).

\section{Une fin de drame romantique}

Je l'avoue, je ne suis pas allé vérifier ni chez Ducéré ni chez Foltzer ni ailleurs. J'ignore si Hugo a lu Bailac, et j'en doute. Je n'ai pas la liste des guides dont il dispose. 
Je suis plein de respect pour les remueurs d'archives et $\mathrm{j}$ 'attends avec espoir qu'ils m'éclairent. Sur les travaux de Napoléon quand il était à Bayonne, sur la malencontreuse baignade de Joséphine, sur la duchesse $\mathrm{C}^{* * *}$, sur le capitaine Lafon - ou Lafont. Les plus récentes informations que j'ai pu recueillir sur le séjour de Napoléon et Joséphine à Bayonne ne m'apportent aucune lumière ${ }^{2}$.

À Bayonne, Victor Hugo revient en arrière, au temps de son enfance, qui est aussi celui d'une épopée fracassée. Son récit est l'occasion d'une double variation sur la mémoire. Une occasion, sans doute autant recherchée que suscitée, de reparcourir un territoire d'enfance, de ressaisir la lumière d'une saison, son éclat unique imprimé à jamais dans l'être. Éclat puissamment réverbérant, fixé sur la plaque de la conscience et de nouveau révélé. Mais le résultat n'est qu'imparfaitement calcographique : le temps ne se rattrape pas. C'est une des marques spécifiques de la relation bayonnaise.

L'enquête est close. Conduite au fil de la lecture, il convient de l'ordonner.

1. Ces pages présentent une unité organique obvie. «Bayonnaises » les définit et les qualifie. Raison pour laquelle je n'aurai garde d'extrapoler. Quant à comparer ces résultats à ceux obtenus ailleurs dans cet «atelier», je ne m'y risque pas non plus : il ne convient pas de croiser des singularités.

2. Le récit de voyage est un genre. Plaisamment, Flaubert - qui est aussi venu à Bayonne avant Hugo - écrit à ce propos :

Le voyageur est tenu de dire tout ce qu'il a vu, le grand talent est de raconter dans l'ordre chronologique : déjeuner au café et au lait, monté en fiacre, station au cours de la borne, musée, bibliothèque, cabinet d'histoire naturelle, le tout assaisonné d'émotions et de réflexions sur les ruines.

Je m'y conformerai donc autant qu'il sera possible (Flaubert, $1987: 20$ ).

Quand le talent vient à manquer, le relateur ne sait que recueillir des « choses misérables », dresser un "relevé de lignes et de surfaces » (Proust, 1989:463). Boileau, en d'autres temps, disait de l'auteur qui n'enfile que des détails :

S'il rencontre un palais, il m'en décrit la face
Et me promène après de terrasse en terrasse.
Il compte du plafond les ronds et les ovales.
Ce ne sont que festons, ce ne sont qu'astragales...
Je saute vingt feuillets pour en trouver la fin
Et me sauve à grands pas à travers le jardin... (v. $51-58 ; 1969: 88)$.

3. Hugo se conforme à sa façon au pacte référentiel lié au récit de voyage. C'est le plus mimétique des genres narratifs. Il donne rarement lieu chez lui à des transcriptions uniquement descriptives : le référentiel, dans ces pages bayonnaises, ne congédie pas le fictionnel. En termes sémiologiques, le récit n’est pas iconique mais indiciel. Il débouche, à un moment ou à un autre, sur le monde intérieur de l'écrivain : le «paysage intérieur» supplante le «paysage extérieur».

${ }^{2}$ Lorblanchès, J.-C., (2009) Napoléon à Bayonne. Paris, L'Harmattan. 
4. En ce sens, ces pages ne sont pas paradoxales : le moi de Hugo s'y répand, tout imprégné qu'il est de Bayonne - de l'Espagne, inséparablement - et, par association, de l'épopée napoléonienne. Toutes ces images fusionnent dans les souvenirs d'enfance rassemblés sur place. Hugo est protéiforme. Son ego le redouble. Égotiste, donc le récit de voyage ? Sans aucun doute. Avec Hugo il n'y a pas à craindre de rapporter l'œuvre à des déterminations personnelles.

5. Des « réminiscences », des « traces » relevées, trouvées ailleurs... Elles sont la conséquence de cette puissance protéiforme. Philippe Murray dit, par calembour, que Hugo n'est pas «avare d'avatars" (1999: 397) mais qu'il est toujours lui-même. Nous avons pu le vérifier: "les verts paradis des amours enfantines» sont différemment peuplés et localisés selon les textes. Toute œuvre vit de ses relations avec d'autres œuvres. Il faut croiser les occurrences avant de s'autoriser à déclarer tel texte matriciel, tel autre second. Et se donner des critères tels que la chronologie des œuvres et surtout leur fonctionnalité stylistique. La rêverie réputée " divagante ", si caractéristique du récit de voyage chez Hugo s'en trouvera mieux analysée. Dans les pages bayonnaises, il est constant qu'elle est téléologique et parfois même didactique, qu'elle a un cap.

6. Souvenirs, mémoire : le Temps. Celui du retour que décrivent ces pages est un temps perdu. La découverte des lieux transporte dans un ailleurs spatio-temporel. Effacées, les traces disparaissent parce que tout s'efface. C'est dans l'ordre, et Hugo ne manifeste pas l'intention de s'en affranchir. Le temps ne lui offre pas d'occasion de retrouvailles. La dominante du voyage, même à Bayonne, n'est pas de retrouver le passé mais de l'évoquer. Victor Hugo n'est pas Marcel Proust.

7. À ce titre, cette évocation est proprement créatrice. Elle recueille, dans leurs successions, les investissements de l'imaginaire, de la sensibilité et de la pensée, au gré des jours, au gré des spectacles comme ferait un journal intime. Elle est appréciable, paradoxalement, à partir de l'écartement entre le descriptible et le décrit. Cet écartement - ou écart en terme stylistique - est doté de positivité et non de négativité. Mesurée à partir de lui, la création peut recevoir son dû.

\section{REFERENCES BIBLIOGRAPHIQUES}

Bailac, J. B., (1827)_Nouvelles chroniques de la ville de Bayonne par un Bayonnais. Bayonne, Duhart-Fauvet.

Boileau, N., (1969) Art poétique, éd. S. Menant. Paris, Garnier-Flammarion.

Daru, É., (1961) « En relisant Victor Hugo : "de Bayonne à Bordeaux, en diligence", les "courriers" d'autrefois ", in Bulletin de la Société de Borda. Vol. 4, pp. 471474.

Flaubert, G., (1987) Par les champs et par les grèves. Genève, Droz.

Gély, C., (1993) La contemplation et le rêve : Victor Hugo, poète de l'intimité. Paris, A.-G. Nizet.

Hugo, V., (1987) Euvres complètes, vol. Voyages. Paris, Bouquins. 
Hugo, V., (1995) Les contemplations. Paris, Flammarion.

Lorblanchès, J.-C., (2009) Napoléon à Bayonne. Paris, L’Harmattan.

Murray, Ph., (1999) Le XIX ${ }^{e}$ siècle à travers les âges. Paris, Gallimard.

Proust, M., (1989) A la recherche du temps perdu. 7, Le temps retrouvé. Paris, Gallimard.

Sainte-Beuve, C.-A., (1885) Nouveaux lundis, tome quatrième. Paris, Calmann Lévy. 